\title{
Intel Scalable System Framework
}

Henry A. Gabb

Intel Corporation

The world depends on high-performance computing (HPC) to solve ever larger scientific, industrial, and societal problems, but we face growing technical and architectural challenges as HPC systems get larger. In traditional HPC, computation, memory/storage, and network performance are becoming more unbalanced so an integrated, holistic approach is needed for future systems. Also, different workloads (e.g., modeling and simulation, scientific visualization, big data analytics, machine learning) stress different parts of the system (compute, memory, I/O). This can lead to divergent, specialized system infrastructures that are dedicated to a particular type of workload. Specialized systems are more expensive to design, build, and manage because they do not benefit from economies of scale. They often require proprietary solutions that can limit software reusability. The solution to this problem requires innovative technologies that are tightly integrated. Intel ${ }^{\circ}$ Scalable System Framework (SSF) provides breakthrough compute, memory/storage, and network performance; a common infrastructure that supports a variety of workloads; standards-based programmability; and broad vendor availability. This is made possible by Intel's broad portfolio of innovative compute, memory and storage, network fabric, and software technologies, which allows unprecedented co-design and system integration. Tighter component integration improves compute density, I/O bandwidth, and network latency while lowering power consumption and overall cost. Intel SSF creates a stable system target for software vendors to help reduce development and maintenance costs. HPC users benefit from a common infrastructure. Reference designs based on Intel SSF help lower entry barriers for equipment manufacturers while still allowing them to innovate. The technical details of each of these high-level Intel SSF features will be discussed. 